\title{
Evaluation of the ECGI Patchwork Method Using Experimental Data in Sinus Rhythm
}

\author{
Oumayma Bouhamama ${ }^{1,2,3}$, Lisl Weynans ${ }^{1,2,3}$, Laura Bear ${ }^{3,4,5}$ \\ ${ }^{1}$ Université de Bordeaux, IMB, UMR5251, Bordeaux, France \\ ${ }^{2}$ CARMEN team, Inria Bordeaux - Sud-Ouest, Bordeaux, France \\ ${ }^{3}$ IHU-Liryc, Fondation Bordeaux Université, Bordeaux, France \\ ${ }^{4}$ INSERM, CRCTB, U1045, Bordeaux, France \\ ${ }^{5}$ Université de Bordeaux, CRCTB, U1045, Bordeaux, France
}

\begin{abstract}
Torso surface and ventricular epicardial potentials were recorded simultaneously in anesthetized, closed-chest pigs $(n=5)$ during sinus rhythm. Activation times were estimated from recorded torso potentials using three classical ECGI methods and a new method called the Patchwork Method (PM), which locally selects the optimal ECGI method and has demonstrated its efficiency with simulated data. The aim of this study was to evaluate the Patchwork method using experimental data in sinus rhythm.

By comparing the classic ECGI reconstructions to recorded epicardial activation mapping, several inaccuracies in the ECGI maps are highlighted in this study. This involved inaccuracies in reconstructing activating maps, locating breakthrough sites and the production of artificial lines of block. However, the PM overcomes these restrictions, demonstrating its abilities to accurately reconstruct activation maps $(C C=0.90[0.86 ; 0.92]$ and $R E=0.20$ $[0.19 ; 0.24])$ and localize epicardial breakthrough sites $(L E=17.16[8.87 ; 22.14])$. Furthermore, it reduced the frequency of artificial lines of block ( 2 of 5 pig hearts).
\end{abstract}

\section{Introduction}

Noninvasive electrocardiographic imaging (ECGI) provides real-time panoramic images of epicardial electrical activity from potential measurements on the torso surface. ECGI is mathematically represented by a Cauchy problem for the Laplace equation:

$\left\{\begin{aligned} \operatorname{div}(\sigma \nabla u) & =0 \text { in the torso volume, } \\ u=u_{T} \text { and } \sigma \nabla u \cdot n & =0 \text { on the torso surface } T,\end{aligned}\right.$

where $u$ is the electric potential and $\sigma$ the conductivity within the torso. Several numerical methods are commonly used for ECGI, including the finite-element method
(FEM), the boundary-element method (BEM) and the method of fundamental solutions (MFS).

Recent validation studies have demonstrated that current implementations are inaccurate in reconstructing electrical activity during sinus rhythm in the presence of conduction abnormalities such as LBBB $[1,2]$ and in structurally heterogeneous hearts [3]. Epicardial breakthrough sites can be misplaced and artificial lines of block, that were not present in recorded maps, could appear. However, there are very few studies that have evaluated ECGI in sinus rhythm.

In a recent study, we described a novel method: the Patchwork Method (PM) and evaluated it using simulated data. This method combines the solutions obtained with FEM and MFS, using the BEM formulation to compare their residuals in order to select the most accurate method [4]. We therefore hypothesize that this PM is more accurate than classical ECGI methods in the reconstruction of activation maps and localisation of breakthrough site, and in particular can reduce the frequency of artificial lines of block in reconstructed activation maps.

The objective of this study was to test this hypothesis and evaluate this new method using experimental data; to assess its ability, and that of standard ECGI methods to accurately reconstruct epicardial electrograms and activation maps.

\section{Patchwork method}

The PM, as has previously been described in [4], locally selects the optimal solution among several classical ECGI methods. According to classical ECGI methods, the linear relationship between cardiac sources and the resulting torso surface potentials can be written in matrix form:

$$
A u_{\mathrm{h}}=u_{\mathrm{T}},
$$

where $A$ is the transfer matrix, $u_{\mathrm{T}}$ represents the unknown cardiac sources, and $u_{T}$ the torso measurements. We de- 
note by $A_{\mathrm{F}}, A_{\mathrm{B}}$ and $A_{\mathrm{M}}$ the transfer matrices obtained with the FEM, BEM and MFS respectively.

Let $u^{\text {ex }}$ be an array representing the exact solution of the inverse problem discretized on an epicardial mesh. Then for all methods the residual of $u^{\text {ex }}$ tends to zero when the mesh size tends to zero:

$$
\lim _{\mathrm{h} \rightarrow 0} R_{\gamma}\left(u^{\mathrm{ex}}\right)=0, \gamma=F, B, M,
$$

where the residual $R_{\gamma}\left(u_{\mathrm{h}}\right)=A_{\gamma} u_{\mathrm{h}}-u_{\mathrm{T}}$. The PM uses this property as a criterion to select locally, among the different numerical solutions, the one that is closest to the exact solution, without knowing what the exact solution is.

In this study, the PM solution is obtained with the FEM and the MFS and a zero-order Tikhonov regularization. For each time step $n$ :

- The approximate solutions $u_{\mathrm{h}, \mathrm{F}}^{n}$ and $u_{\mathrm{h}, \mathrm{M}}^{n}$ are computed, - These solutions are used to compute the forward solution and the associated residuals $R_{\mathrm{B}}\left(u_{\mathrm{h}, \mathrm{F}}^{n}\right)$ and $R_{\mathrm{B}}\left(u_{\mathrm{h}, \mathrm{M}}^{n}\right)$ using a BEM formulation,

- For each epicardial point, a coefficient $\alpha^{n}$ is defined. Its value is 0 if the smallest residual on the nearest torso point is obtained with the MFS, and 1 otherwise.

A temporal regularization of the coefficient $\alpha$ is performed to avoid sudden variations between successive time steps. For each time step $n$, the new approximate solution is

$$
u_{\mathrm{h}}^{n}=\alpha^{n} u_{\mathrm{h}, \mathrm{F}}^{n}+\left(1-\alpha^{n}\right) u_{\mathrm{h}, \mathrm{M}}^{n} .
$$

\section{Experimental Data and Evaluation Metrics}

ECGI reconstructions using the PM, FEM, MFS and BEM methods were evaluated using an in-vivo experimental pig data set that has previously been described [5]. All surgical procedures were approved by the Animal Ethics Committee of the University of Auckland and conform to the Guide for the Care and Use of Laboratory Animals (National Institutes of Health publication No. 85-23).

Briefly, a midline sternotomy was performed on 5 closed-chest pigs (30-40 kg) under anesthesia. A 239 electrode sock (5-10 mm spacing) was drawn over the ventricles, then the thorax closed and air expelled. Flexible strips (BioSemi, the Netherlands) with 184 electrodes (30-45 $\mathrm{mm}$ spacing) were attached to the body surface. Epicardial and body surface potentials were recorded simultaneously at $2 \mathrm{kHz}$ during sinus rhythm. At the end of each experiment, the heart was arrested with potassium citrate, and magnetic resonance (MR) images of the heart and thorax were acquired. The heart was excised and epicardial electrode locations were captured with a multi axis digitizing arm (FARO Technologies, FL). MR imaging contrast markers placed on the sock and body surface strips were localized in the MR images and used to register electrode locations.
Activation times were derived from recorded sock and ECGI electrograms as the time of minimum derivative $(\mathrm{dV} / \mathrm{dt})$ over the QRS. Correlation coefficients (CC) and relative errors (RE) were computed between true and inverse-computed activation times. Epicardial breakthrough sites were identified from activation maps as sites with a local minimum in activation time. The centers of the early activation areas were taken as the breakthrough sites. The localization error (LE) of these breakthrough sites was calculated using the geodesic distance, and the time difference between the ECGI-recorded and the nearest simulated breakthroughs was calculated. Lines of conduction block were automatically computed from activation maps from pig heart as a local activation time jump of $20 \mathrm{~ms}$ or more between contiguous electrodes [2]. Statistical analyses were performed using GraphPad Prism 8.3. For each metric, the significance of the differences was tested using paired $t$-tests with $p<0.05$ defined as significant. Data are expressed as median with interquartile range.

\section{Results and Discussion}

Figure 1 shows two different activation maps in hearts in normal sinus rhythm reconstructed with the MFS, FEM, BEM and PM (Panel A and C). The ventricles are shown in anterior and posterior view respectively. Panels A and $\mathrm{C}$ show a smooth propagation of the activation wavefront for recorded maps. However, the reconstructed activation maps using classical ECGI method demonstrate a long line of block across the septum between the ventricles in both hearts. The activation map reconstructed by PM was substantially smoother and lines of block were not present (panel C) or shorter (panel A), as was reflected by the improvements in $\mathrm{CC}$ and $\mathrm{RE}$.

By analyzing the reconstructed electrograms (Panel B), it can be seen that the lines of blocks arise due to an inaccuracy in the reconstruction of electrograms. That is, classical methods reconstruct electrograms with a "W" shape during the QRS so that small changes in the voltage can cause the activation marker to jump quickly between the two downslopes and produce this artificial line. Previous studies have also seen this phenomenon $[4,6]$ and suspect that the appearance of $\mathrm{W}$-shaped electrograms results from the reconstructed potentials representing a distant field of both the epicardial and endocardial electrical activity and not a purely local epicardial activation. These $\mathrm{W}$-shaped electrograms are less apparent in the PM reconstructions, and hence the activation wavefront travels more smoothly across the epicardial surface, similar to the recorded case.

In Panel A, we also present the true (white spheres) and reconstructed (grey spheres) breakthrough sites detected using each of the different methods. In this example, the MFS localized breakthrough sites better than the BEM and the FEM. The PM provided the more accurate localization 
A Recorded AT
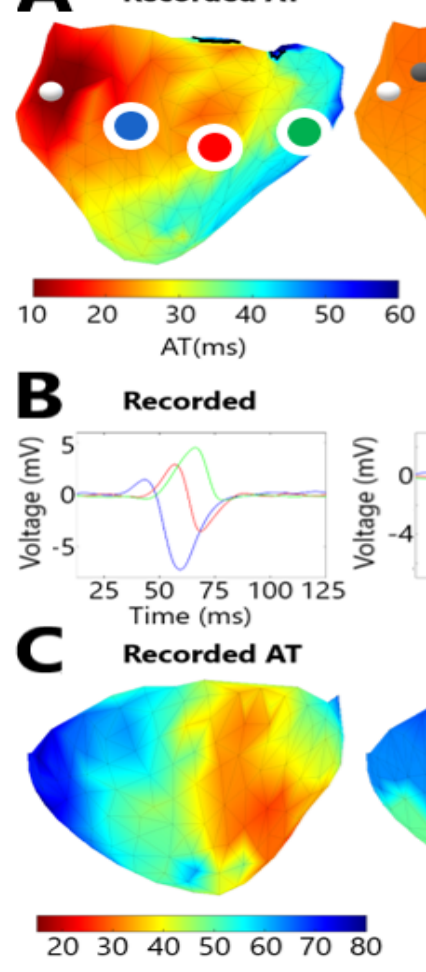

AT (ms)
MFS

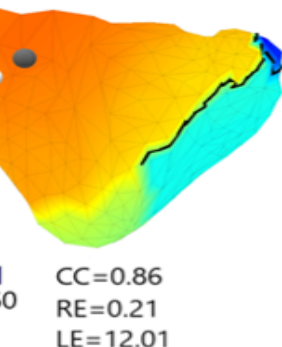

MFS

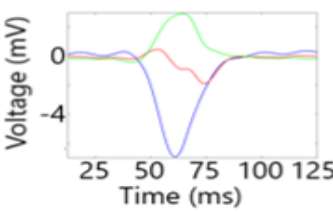

MFS

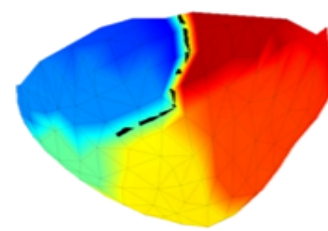

$\mathrm{CC}=0.88$

$\mathrm{RE}=0.19$

$L E=31.87$
FEM

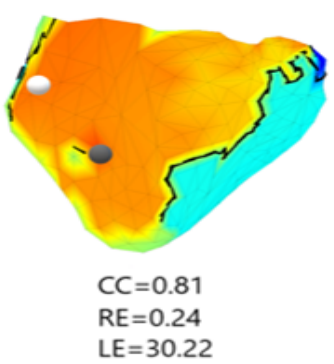

FEM

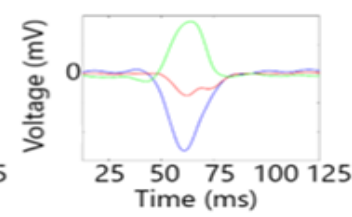

FEM

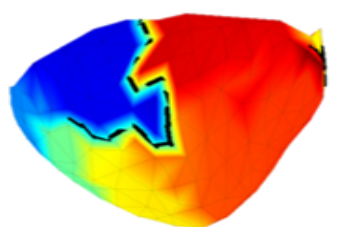

$C C=0.89$

$\mathrm{RE}=0.19$

LE $=32.17$
BEM

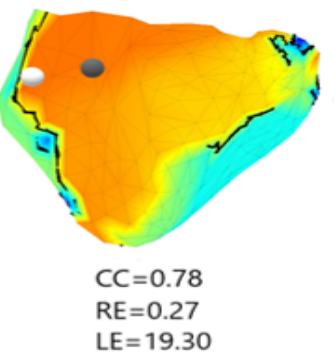

BEM

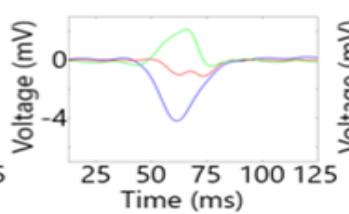

BEM

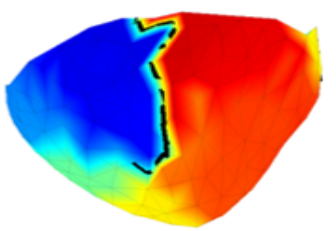

$\mathrm{CC}=0.77$

$\mathrm{RE}=0.31$

$\mathrm{LE}=32.74$

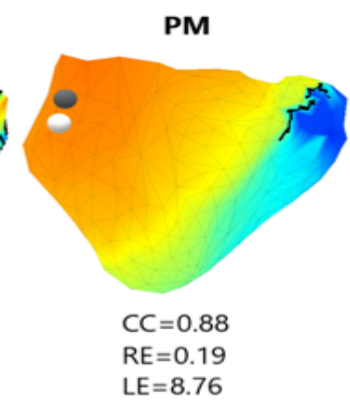

PM

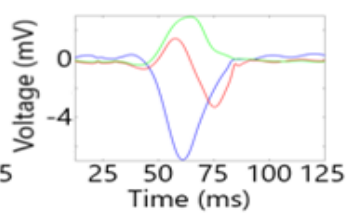

PM

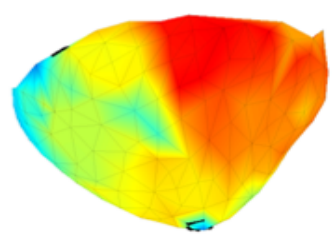

$C C=0.91$

$\mathrm{RE}=0.18$

$L E=25.19$

Figure 1: Recorded and ECGI-reconstructed activations maps in normal sinus rhythm (A and C), demonstrate the smooth activation seen in recorded maps compared to the distinct lines of block (black lines) often seen in ECGI reconstructions (A) (white: recorded ;grey: reconstructed epicardial breakthrough site). (B) Recorded and reconstructed ECGI Electrograms at colour-coded electrodes marked on the recorded activation map of (A).
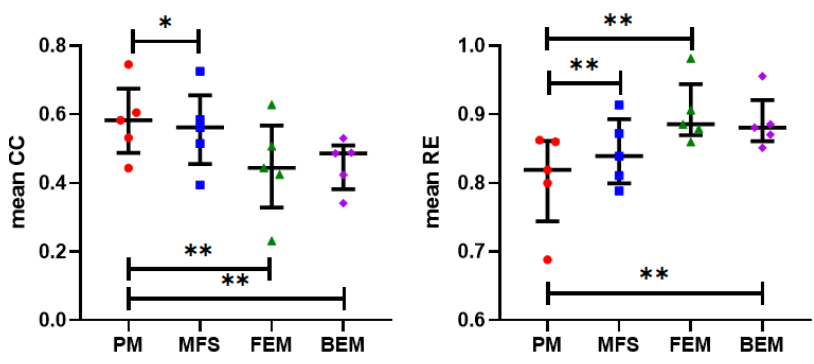

Figure 2: Mean CC and mean RE for sinus rhythm for the PM, MFS, FEM and BEM

of this breakthrough site ( $\mathrm{LE}=8.76 \mathrm{~mm})$ compared to the MFS $(\mathrm{LE}=12.01 \mathrm{~mm})$, FEM $(\mathrm{LE}=30.22 \mathrm{~mm})$ and BEM $(\mathrm{LE}=19.30 \mathrm{~mm})$,

For each sinus beat, the mean CC and the mean RE for potentials were computed over all nodes. Figure 2 presents a comparison of the mean CC and mean RE of the different methods across all sinus rhythm data. Potentials reconstructed with the PM were more correlated to the measured potentials than those reconstructed by the MFS, FEM or the BEM $(p<0.05)$. PM also reduced the RE for electrograms $(p<0.05)$.

Figure 3 shows the CC, RE between activation maps, and the LE of breakthrough sites for each method for the 5 pigs. As with potentials, activation maps reconstructed by PM showed a higher correlation to the ground truth activation maps than those reconstructed by MFS, FEM and BEM alone $(p=0.04)$. PM also reduced the RE for activation maps $(p=0.006)$.

Though all ECGI methods succeeded in capturing the general location of the breakthrough sites, the LE obtained by the PM was substantially smaller $(\mathrm{LE}=17.16[8.87$ ; 22.14]) than that obtained by standard ECGI methods $(\mathrm{LE}=26.84$ [15.97; 31.41] for MFS, 30.22 [23.40 ; 31.66] for FEM and 30.52 [21.44; 32.94] for BEM). The time differences between the nearest actual breakthroughs and ECGI-detected breakthroughs acquired by the PM were also smaller than by the other methods.

This study has demonstrated that, when using PM, the potentials and the activation maps are more correlated 

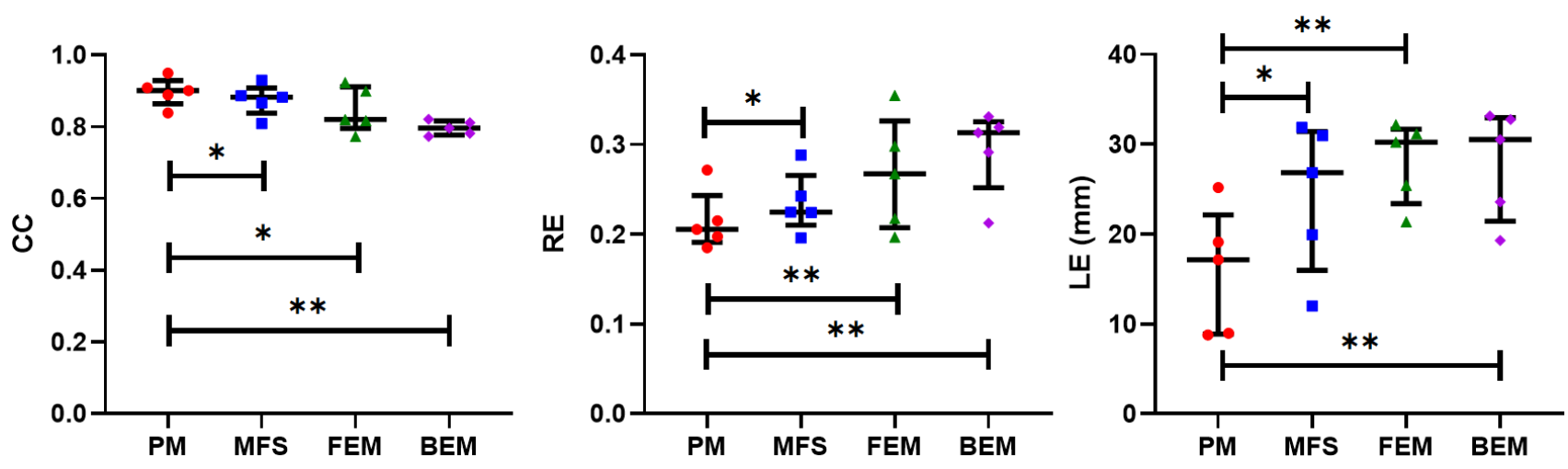

Figure 3: The CC, RE and LE values for activation maps for sinus rhythm for the PM, MFS, FEM and BEM.

to those recorded than those reconstructed with classical methods such as FEM, MFS and BEM. Also the estimated reconstruction error is minimized. Furthermore, the localization of breakthrough sites is more accurate using PM than MFS, FEM and BEM. Though lines of block, that were absent in recorded maps, were present across all reconstructed activation maps using the MFS, FEM or BEM, these artificial lines were identified in only 2 of 5 pig hearts activation maps reconstructed by the PM.

In the future work, we intend to validate the Patchwork Method using clinical data. Though results will surely not be better than in the case of simulation and experimental data, due to geometric errors and other uncertainties, we expect that the PM will improve the reconstruction of the electrical potentials and activation times on the heart, as it selects the optimal method in each area and at each time step. Also, we aim to improve the results by combining different regularization methods with the PM.

\section{Conclusion}

The novel Patchwork Method demonstrates a higher level of accuracy in reconstructing activation maps and in locating breakthrough sites in sinus rhythm for experimental data than classical methods. Importantly, these improvements are also seen in the reduced frequency of artificial lines of block.

\section{Acknowledgments}

This work was supported by the French National Research Agency (ANR-10-IAHU04-LIRYC). This work was granted access to HPC resources of CINES under GENCI allocation 2020-A0070307379.

\section{References}

[1] L. R. Bear et al. Cardiac electrical dyssynchrony is accurately detected by noninvasive electrocardiographic imaging. Heart Rhythm, 15:1058-1069, 2018.

[2] J. Duchateau et al. Performance and limitations of noninvasive cardiac activation mapping. Heart Rhythm, 16:435-442, 2018.

[3] J. L. Sapp et al. Inverse solution mapping of epicardial potentials: quantitative comparison with epicardial contact mapping. Circ. Arrhythm. Electrophysiol., 5:1001-1009, 2012.

[4] O. Bouhamama et al. A Patchwork Inverse Method in Combination with the Activation Time Gradient to Detect Regions of Slow Conduction in Sinus Rhythm. Computing in Cardiology, 2020-September:1-5, 2020.

[5] L. R. Bear et al. Forward Problem of Electrocardiography: Is It Solved? Circulation: Arrhythmia and Electrophysiology, 8(3):677-684, 2015.

[6] L. Bear et al. Advantages and pitfalls of noninvasive electrocardiographic imaging. J. Electrocardiol., 57:15-20, 2019.

Address for correspondence:

Oumayma Bouhamama

Inria Bordeaux - Sud-Ouest

200 Avenue de la Vieille Tour, 33405 Talence, Bordeaux, France oumayma.bouhamama@u-bordeaux.fr 